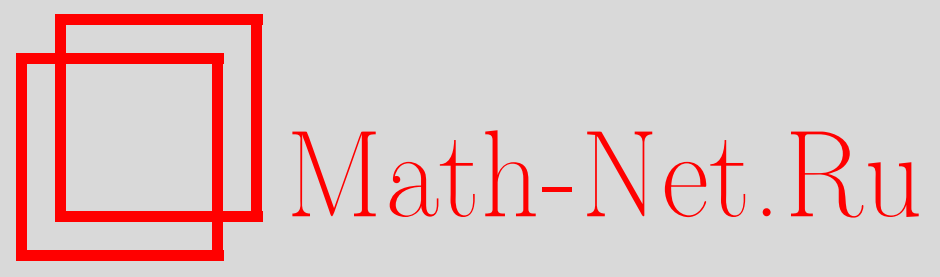

П. Гаврон, В. В. Некрашевич, В. И. Сущанский, Классы сопряженности группы автоморфизмов дерева, Матем. заметки, 1999, том 65, выпуск 6, 938-941

DOI: https://doi.org/10.4213/mzm1131

Использование Общероссийского математического портала Math-Net.Ru подразумевает, что вы прочитали и согласны с пользовательским соглашением http://www. mathnet.ru/rus/agreement

Параметры загрузки:

IP : 54.198 .55 .26

26 апреля 2023 г., $16: 42: 40$

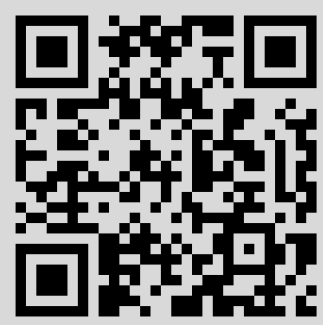




\section{КЛАССЫ СОПРЯЖЕННОСТИ ГРУППЫ АВТОМОРФИЗМОВ ДЕРЕВА}

\section{П. Гаврон, В.В. Некрашевич, В. И. Сущанский}

1. Введение. После работы Титса [1] исследования групп автоморфизмов деревьев различного типа проводились в многочисленых работах различных авторов. Например, в [1] Титс исследовал нормальное строение группы автоморфизмов дерева. Нормальное строение группы автоморфизмов корневого дерева описано в [2] и [3]. Знойко [4] доказал, что группы автоморфизмов регулярных деревьев совершенны, Моллер [5] охарактеризовал их максимальные подгруппы. Действия свободных групп и амальгамированных свободных произведений на деревьях исследовались в [6]. Некоторые проблемы о группах автоморфизмов дерева были поставлены Линдоном в [7].

В ряде работ исследовались классы сопряженности в группах автоморфизмов дерева. В [4] доказано, что все отражения порядка 2 сопряжены в группе автоморфизмов дерева. Критерий сопряженности для автоморфизмов корневого сферически однородного дерева сформулирован в [8]. В [3] доказано, что все сферически транзитивные автоморфизмы сферически однородного корневого дерева сопряжены между собой.

Полная система представителей классов сопряженности в группе автоморфизмов корневого бинарного дерева построена в [9]. В [10] доказано, что группа автоморфизмов регулярного дерева является амбивалентной, т.е. любой ее элемент сопряжен со своим обратным.

В данной заметке мы дадим полное описание классов сопряженности автоморфизмов локально конечного дерева и локально конечного корневого дерева.

Все ранее известные характеризации класов сопряженности являются следствиями нашего описания.

2. Предварительные сведения. Пусть $V=\mathrm{V} T$ - множество вершин (неориентированного) дерева $T, E=\mathrm{E} T$ - множество его ребер. Через $\{a, b\}$ мы будем обозначать ребро, соединяюшее вершины $a, b$. Вершины $a, b$ сме сные, если $\{a, b\} \in E$. Последовательность попарно различных вершин $\left(a_{1}, \ldots, a_{n}\right)$ таких, что $\left\{a_{i}, a_{i+1}\right\} \in \mathrm{E} T$ для всех $i=1, \ldots, n-1$, называется nymeм в дереве $T$, соединяющим вершины $a_{1}$ и $a_{n}$. Для каждых двух вершин $x, y \in \mathrm{V} T$ существует единственный путь $\Pi(x, y)$, соединяющий $x$ и $y$. Функция $d(x, y)=|\Pi(x, y)|, x, y \in V$, где $|\Pi|-$ длина пути (число ребер), является метрикой на $V$. В бесконечных деревьях можно рассматривать пути, бесконечные в одну или в обе стороны. Бесконечный в одну сторону путь $\left(a_{0}, a_{1}, a_{2}, \ldots\right)$ назьвается лучем в дереве $T$, а последовательность $\left(\ldots, a_{-1}, a_{0}, a_{1}, \ldots\right)$, где $\left\{a_{i}, a_{i+1}\right\} \in \mathrm{E} T$, $i \in \mathbb{Z}$, называется прямой в $T$. На каждой прямой можно задать две ориентации. Прямая с фиксированной ориентацией называется ориентированной.

Подстановка $\pi$ множества VT является ав томорфизмом дерева $T$, если она сохраняет отношение смежности вершин на $T$, т.е. если для любых $x, y \in \mathrm{V} T$ из $\{x, y\} \in \mathrm{E} T$ следует $\left\{x^{\pi}, y^{\pi}\right\} \in \mathrm{E} T$.

Группу всех автоморфизмов дерева $T$ будем обозначать через Aut $T$.

Лемма 1 [1]. Пусть $u \in \operatorname{AutT.~Тогда~справедливо~одно~и~только~одно~из~следующих~}$ утверждений:

Работа выполнена при частичной поддержке фонда INTAS, грант № 94-3420. 
1) существует $a \in \mathrm{VT}$ такая, что $a^{u}=a$;

2) существует единственное ребро $\{a, b\} \in \mathrm{ET}$ такое, что $a^{u}=b u b^{u}=a$;

3) существует единственная прямая $L$ дерева $T$ такая, что $L^{u}=L$ и ограничение $\left.u\right|_{L}$ автоморфизма и на $L$ является сдвигом, т.е. при некотором натуральном $d_{0}$ для всех $a \in L$ имеет место равенство $d\left(a, a^{u}\right)=d_{0}$.

Автоморфизмы типа 1), 2) и 3) называются вращениями, отражсениями и сдвигами соответственно. Каждый сдвиг индуцирует естественным образом ориентацию на инвариантной прямой направлением сдвига.

Пусть $G<\operatorname{Aut} T$-подгруппа группы автоморфизмов дерева $T, \bar{V}=V / G$-множество ее орбит при действии на вершинах, а $\bar{E}=E / G$ - множество ее орбит при действии на ребрах дерева $T$. Определим граф̆ орбит $T / G$ следуюшим образом. Вершинами этого граффа являются элементы $\bar{V}$, а две орбиты $\bar{x}, \bar{y}$ соединены ребром $\bar{e}$ в $T / G$ тогда и только тогда, когда существуют представители $x \in \bar{x}, y \in \bar{y}, e \in \bar{e}$ такие, что $e=\{x, y\}$. Заметим, что графф $T / G$, вообще говоря, не является симплициальньм, т.е. может иметь кратные ребра и петли.

В частности, графф орбит циклической группы $\langle u\rangle$, порожденной автоморфизмом $u \in$ Aut $T$, обозначим через $T_{u}$ и назовем графом орбит автоморфизма $u$. Орбитальным типом автоморфизма $u$ назьвается граф орбит $T_{u}$ с вершинами, помеченными кардинальными числами, равньми мощности соответствующих орбит. Орбитальньй тип автоморфизма $u$ будем обозначать через $T(u)$. Два орбитальных типа эквивалентны, если они изоморфны как помеченные деревья, т.е. если существует их изоморфизм как графов, сохраняющий метки вершин.

Группа Aut $T$ действует на регулярном дереве $T$ импримитивно. Единственной нетривиальной системой импримитивности Aut $T$ на множестве VT является разбиение на классы эквивалентности “ ", заданной условием

$$
x \sim y \Longleftrightarrow d(x, y) \equiv 0(\bmod 2) .
$$

Это разбиение состоит из двух классов $V_{0}$ и $V_{1}$. Подгруппа $(\operatorname{Aut} T)^{+}<\operatorname{Aut} T$ автоморфизмов, сохраняющих множества $V_{0}, V_{1}$, имеет индекс 2 в группе Aut $T$ и является простой [1].

Сдвиг принадлежит подгруппе $(\mathrm{Aut} T)^{+}$тогда и только тогда, когда он сдвигает инвариантную прямую на четное расстояние.

Дерево $T$ с фиксированной вершиной $x_{0}$ называется корневым деревом, а вершина $x_{0}-\kappa o p$ немэтого дерева. Множество вершин корневого дерева естественным образом распадается в дизъюнктное объединение $V=\bigcup_{k \geqslant 0} V_{k}$, где $V_{k}=\left\{x: d\left(x_{0}, x\right)=k\right\}$. Множество $V_{k}$ назьвается $k$ сферой (слоем) корневого дерева $\left(T, x_{0}\right)$.

Автоморфизмами корневого дерева $\left(T, x_{0}\right)$ являются автоморфизмы дерева $T$, фиксирующие вершину $x_{0}$. Следовательно, группа автоморфизмов $\operatorname{Aut}\left(T, x_{0}\right)$ корневого дерева $\left(T, x_{0}\right)$ совпадает со стабилизатором вершины $x_{0}$ в группе автоморфизмов дерева $T$ :

$$
\operatorname{Aut}\left(T, x_{0}\right)=\operatorname{St}_{\mathrm{Aut} T}\left(x_{0}\right) \text {. }
$$

Корневое дерево называется однородным на сфере $k$, если степени всех его вершин $k$-й сферы равны. Дерево называется сферически однородным, если оно однородно на каждой сфере. Сферически однородное дерево однозначно определяется своим сферическим индексом- последовательностью чисел $\left[n_{0}, n_{1}, n_{2}, \ldots\right]$, где $n_{k}$ - число ребер, соединяющих вершину $k$-й сферы с вершинами $(k+1)$-й сферы. С ферически однородное корневое дерево сферического индекса $[n, n, \ldots]$ назьвается $n$-регулярным корневым деревом, или просто регулярным корневым деревом.

\section{3. Классы сопряженности в группе автоморфизмов корневого дерева.}

ЛЕмма 2. Для любой әруппь автоморфизмов $G \leqslant \operatorname{Aut}\left(T, x_{0}\right)$ граф орбит $T / G$ является деревом. В частности, граф орбит произвольного автоморфизма корневого дерева является деревом.

Введенное в п. 2 понятие орбитального типа позволяет сфформулировать критерий сопряженности в группе автоморфизмов корневого дерева, являющийся обобщением критерия сопряженности подстановок в симметрической группе. 
Tеорема 1. Автоморфизмы корневого дерева $\left(T, x_{0}\right)$ сопряжены в әруппе $\operatorname{Aut}\left(T, x_{0}\right)$ тогда и только тогда, когда их орбитальные типь әквивалентны.

Будем говорить, что автоморфизм $u \in \operatorname{Aut}\left(T, x_{0}\right)$ является сферически транзитивным, если цик лическая группа $\langle u\rangle$ действует транзитивно на каждой сфере дерева $\left(T, x_{0}\right)$.

СлЕдСтвиЕ 1 [3]. Произвольные два сферически транзитивных автоморфизма корневого дерева $\left(T, x_{0}\right)$ сопряхены в $\operatorname{Aut}\left(T, x_{0}\right)$.

Группа $G$ называется амбивалентной, если любой ее элемент сопряжен со своим обратным.

СлеДСтвиЕ 2 [10]. Для любого корневого дерева $\left(T, x_{0}\right)$ әруппа $\operatorname{Aut}\left(T, x_{0}\right)$ является амбивалентной.

4. Сопряженность в группе автоморфизмов некорневого дерева. Пусть $T$ - произвольное дерево. Ясно, что автоморфизм, сопряженный с вращением (отражением, сдвигом), также является вращением (отражением, сдвигом соответственно). Поэтому можно описывать классы сопряженности в Aut $T$ отдельно для каждого случая.

Вращение $u$ (некорневого) дерева $T$ можно рассматривать как автоморфизм корневого дерева $\left(T, x_{0}\right)$, где $x_{0}$ - неподвижная точка автоморфизма $u$. Орбитальным типом этого автоморфизма (корневого дерева) является помеченное корневое дерево. Оно определяет помеченное дерево (без фиксации корневой вершины), которое назовем орбитальным типом вращения $и$. Определенный таким образом орбитальный тип автоморфизма не зависит от выбора неподвижной точки $x_{0}$. Соответственно два орбитальньх типа вращений некорневого дерева называются әквивалентными, если они изоморфны как помеченные деревья.

Теорема 2. Вращения $u, v \in \operatorname{Aut} T$ сопряженьи в $\operatorname{Aut} T$ тогда и только тогда, когда их орбитальные типь әквивалентны.

Пусть $u$ - отражение дерева $T$ и $x_{0}, y_{0}$ - смежные вершины такие, что $x_{0}^{u}=y_{0}$ и $y_{0}^{u}=x_{0}$. Построим новое дерево $T^{*}$, присоединив к VT новую вершину $p$. Соединим $p$ с вершинами $x_{0}, y_{0}$ и удалим ребро $\left\{x_{0}, y_{0}\right\}$. Действие автоморфизма $u$ на множестве вершин дерева $T$ можно продолжить до $T^{*}$, полагая $p^{u}=p$; при этом полученное действие будет автоморфизмом корневого дерева $\left(T^{*}, p\right)$.

Орбитальным типом отражения $u$ дерева $T$ назовем орбитальный тип автоморфизма $u$ корневого дерева $\left(T^{*}, p\right)$.

Теорема 3. Отражения $u, v \in$ Aut $T$ сопряжень в группе Aut $T$ тогда и только тогда, когда их орбитальные типы эквивалентны.

ЗАмечАниЕ. Знойко [4] установил, что все отражения порядка 2 в группе автоморфизмов регулярного дерева сопряжены. Все вершины орбитальных типов (кроме корневой) для таких автоморфизмов помечены числом 2 , и несложно заметить, что они эквиваленты.

Смещ, ением автоморфизма $u \in \operatorname{Aut} T$ назовем число $d(u)=\min _{x \in T}(x, d(x))$.

Tеорема 4. Пусть $u, v \in \operatorname{Aut} T-$ сдвиги с инвариантными (ориентированными направлением сдвига) прямыми $L_{1}, L_{2}$. Автоморфизмы $u, v$ сопряжены в группе Aut $T$ тогда и только тогда, когда $L_{1}, L_{2}$ принадлежат одной орбите действия Аut $T$ на множестве ориентированных прямых дерева $T$ и смещения $d(u)$ и $d(v)$ равны.

Так как группа автоморфизмов регулярного дерева $T$ действует на множестве ориентированньх прямых транзитивно, из теоремы 4 получаем

СлЕдСтвиЕ 3. Сдвиги $u, v$ регулярного дерева $T$ сопряжсены тогда и только тогда, когда $d(u)=d(v)$.

Из сформулированных выше теорем 2-4 вытекает

СледСтвиЕ 4. Для любого регулярного дерева $T$ его группа автоморфизмов является амбивалентной. 
5. Сопряженность в $(\text { Aut } T)^{+}$для случая регулярного дерева. Пусть $T$-регулярное дерево. Группа $(\mathrm{Aut} T)^{+}$состоит из всех вращений и всех сдвигов с четным смещением. Поскольку дерево $T$ регулярное, $(\text { Aut } T)^{+}$действует на множестве ориентированных прямых дерева $T$ транзитивно.

Теорема 5. Сдвиги дерева $T$, принадлежсащие группе $(\text { Aut } T)^{+}$, сопрялсень в $(\text {Aut } T)^{+}$ тогда и только тогда, когда их смещения равны.

Два вращения $u, v$ дерева $T$ сопряжены в Aut $T$ тогда и только тогда, когда существуют две вериины $x_{0}, y_{0}$ дерева $T$, лехсащие в одном блоке импримитивности, такие, что $x_{0}$ - неподвижная точка автоморфизма $u$, а $y_{0}$ - неподвижная точка автоморфизма $v$, и орбитальные типь $u, v$ как автоморфизмов корневых деревьев $\left(T, x_{0}\right) u\left(T, y_{0}\right)$ соответственно эквивалентны.

\section{СПИСОК ЦИТИРОВАННОЙ ЛИТЕРАТУРЫ}

1. Tits J. // Essays on Topology and Related Topics. Mémoirie dédies á G. de Rham. Berlin: Springer, 1980. Р. 188-211. 2. Сущанский В. И. // Бесконечные группы и примькающие алгебраические структуры. Киев: ИМ АН Украины, 1993. С. 269-289. 3. Bass Н., Otero-Espinar M.V., Rockmore D. N., Tresser C. P. L. Cyclic Renormalisation and Automorphism Groups of Rooted Trees. Berlin: Springer, 1996. 4. Знойко Д. В. // Матем. сб. 1977. Т. 32. № 1. C. 109-115. 5. Möller R. // J. London Math. Soc. (2). 1991. V. 43. P. 236-252. 6. Serre J.-P. Trees. New York: Springer, 1988. 7. Lyndon R. // Combinatorial Groups Theory and Topology / ed. S. M. Gersten, J. R. Stallings. Ann. of Math. Stud. Princeton: Princeton Univ. Press, 1987. P. 3-33. 8. Безущак О.Е., Сущанский В. И. // Укр. матем. ж. 1991. Т. 43. № 7-8. С. 1031-1038. 9. Brunner A. M., Sidki S. // J. Algebra. 1997. V. 195. Р. 465-486. 10. Сущанский В. И. // Доп. АН України. 1992. Т. 11. С. 5-9.

Institute of Mathematics, Silesian Technical University

E-mail: pgawron@zeus.polsl.gliwice.pl, nazaruk@ukrpack.net, wsusz@zeus.polsl.gliwice.pl 